\title{
Edge-Markovian Dynamic Graph Based Information Dissemination Model for Mobile Social Networks
}

\author{
Li Qiu ${ }^{\dagger}$, Yong $\mathrm{Li}^{\dagger}$, Pan Hui ${ }^{\ddagger}$, Li Su ${ }^{\dagger}$ \\ $\dagger$ Department of Electronic Engineering, Tsinghua University, Beijing 100084, China \\ $\ddagger$ Deutsche Telekom Laboratories/TU-Berlin, Ernst-Reuter-Platz 7, Berlin 10587 \\ yong-li07@mails.tsinghua.edu.cn
}

\begin{abstract}
In this poster, we apply Edge-Markovian Dynamic Graphs to present an analysis framework to evaluate the average delay for the information dissemination in Mobile Social Networks. It is the first model to give a detailed expression of average information dissemination delay based on message size, transmission willingness and other parameters. Extensive simulation results reveal the influence of those parameters.
\end{abstract}

\section{INTRODUCTION}

Mobile Social Networks have attracted increasing attention in recent years, due to the proliferation of smart phones and online social networking services. A fundamental problem in such kind of network is how fast the information can propagate among the users. To solve it, we model the MSN as numerous nodes forming a graph where every single node represents a mobile phone and the link between two nodes exists if they are connected (i.e. each of the two nodes can send a message to the other one), which is called edge-Markovian dynamic graph. We derive the information dissemination delay of MSN. Our main contribution is a closedform expression of the information dissemination delay as a function of message size, ratio of infected nodes, user's selfishness, and the involved number of users.

\section{SYSTEM MODEL}

We divide all the nodes in the graph into two nonoverlapping communities A and B based on their distinct location properties and professional affiliation. We assume there are $N$ nodes in $\mathrm{A}, M$ nodes in $\mathrm{B}$, and a node in A gets the information initially. Every link in

Permission to make digital or hard copies of all or part of this work for personal or classroom use is granted without fee provided that copies are not made or distributed for profit or commercial advantage and that copies bear this notice and the full citation on the first page. To copy otherwise, to republish, to post on servers or to redistribute to lists, requires prior specific permission and/or a fee.

ACM CoNEXT Student Workshop, December 6, 2011, Tokyo, Japan.

Copyright 2011 ACM 978-1-4503-1042-0/11/0012 ...\$10.00. the graph has contact and inter-contact periods, and we assume every contact period as well as inter-contact period follows an exponential distribution with intensity $\mu$ and $\lambda$ respectively. We define intra-group link generation (perish) speed as $\lambda_{1}\left(\mu_{1}\right)$ and inter-group generation (perish) speed as $\lambda_{2}\left(\mu_{2}\right)$. Usually $\lambda_{1}$ is much larger than $\lambda_{2}$. We assume all the links, when up, have equal capacity. We refer to $\alpha$, which represents the time needs to transmit a certain message as its bundle size. According to exponential law's character, the probability of a contact period being greater than $\alpha$ can be calculated by the integral of $\int_{\alpha}^{+\infty} \mu e^{-\mu t} d t=e^{-\mu \alpha}$.

Therefore, on average a message with size $\alpha$ is transmitted with probability $e^{-\mu \alpha}$ through a link once the link comes into existence, in other words after a link has been connected for $1 / e^{-\mu \alpha}$ times, the message has a high probability of being transmitted. It thus could be interpreted as the link generating speed decreases to $\lambda e^{-\mu \alpha}$, or $\lambda_{1} e^{-\mu_{1} \alpha}$ for intra-group link generating speed and $\lambda_{2} e^{-\mu_{2} \alpha}$ for inter-group link generating speed in our model. We assume on average the intra-group and intergroup probability of people willing to forward a message is equal to $p_{1}$ and $p_{2}$ respectively. Consequently, the intra-group speed changes to $p_{1} \lambda_{1} e^{-\mu_{1} \alpha}$ while the inter-group speed changes to $p_{2} \lambda_{2} e^{-\mu_{2} \alpha}$.

\section{INFORMATION DISSEMINATION DELAY}

In this poster, we focus on the average delay for a certain percentage of nodes in A being infected. We refer to $r$ as the preset percentage which is greater than 0 and less than 1 . Assuming $i$ stands for the number of nodes being infected in A and $j$ for B. According to the definition above, the Markov chain has the following $(\lceil r * N\rceil-1) *(M+1)+1$ distinct states: transient state $(i, j)$, where $1 \leq i \leq\lceil r * N\rceil-1$ and $0 \leq j \leq M$; absorbing state Succ, which means at least $r * N$ nodes in A have been infected.

Given two sets of nodes $\mathrm{U}$ and $\mathrm{W}$, if every node in $\mathrm{U}$ is able to infect each node in $\mathrm{W}$ with probability $p$, then the odds that $m$ nodes in $\mathrm{W}$ will be infected is computed as follows [2]:

$$
P_{\mathrm{inf}}(m, p,|U|,|W|)=C_{|W|}^{m}(1-p)^{|U|(|W|-m)}\left(1-(1-p)^{|U|}\right)^{m} .
$$




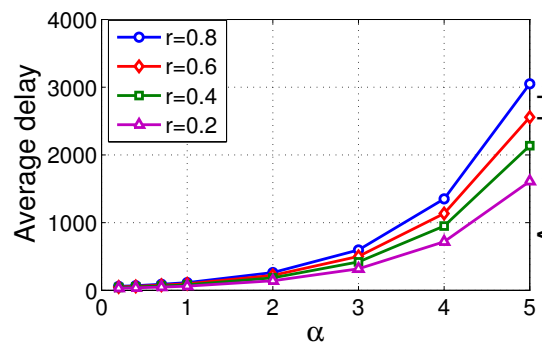

(a) Size of message

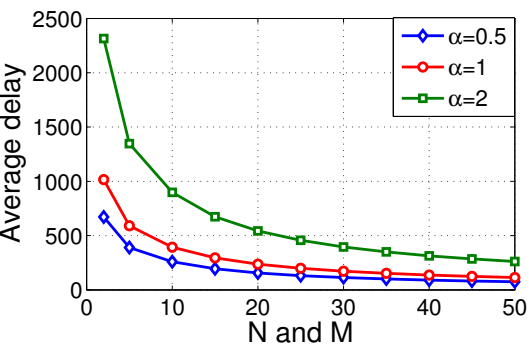

(b) Number of nodes

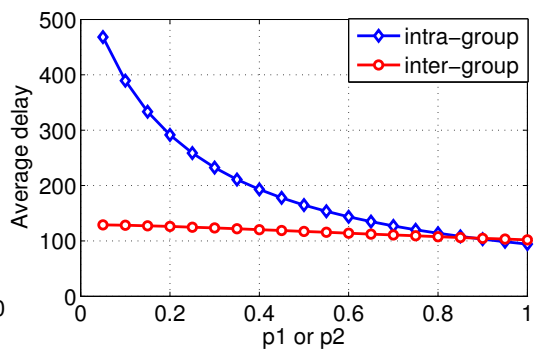

(c) Transmission willingness

Figure 1: The influence of various parameters on average information dissemination delay.

We define $P_{i, j \rightarrow \text { succ }}$ as the probability of shifting from the present state $(i, j)$ to the state Succ; define $P_{i, j \rightarrow i^{\prime}, j^{\prime}}$ as the transition probability from present state $(i, j)$ to $\left(i^{\prime}, j^{\prime}\right)$. Such two probabilities are functions of time $t$, when $t$ is relatively small, it can be calculated in the following integrals:

$$
\begin{aligned}
& P_{i, j \rightarrow s u c c}= \sum_{n=\lceil r * N\rceil}^{N} \sum_{m=0}^{n-i} P_{\inf }\left(m, p_{1} \lambda_{1} e^{-\mu_{1} \alpha} t, i, N-i\right) \\
& \times P_{\inf }\left(n-i-m, p_{2} \lambda_{2} e^{-\mu_{2} \alpha} t, j, N-i-m\right) \\
& P_{i, j \rightarrow i^{\prime}, j^{\prime}}= \sum_{m=0}^{i^{\prime}-i j^{\prime}-j} \sum_{n=0} P_{\inf }\left(m, p_{1} \lambda_{1} e^{-\mu_{1} \alpha} t, i, N-i\right) \\
& \times P_{\inf }\left(i^{\prime}-i-m, p_{2} \lambda_{2} e^{-\mu_{2} \alpha} t, j, N-i-m\right) \\
& \times P_{\inf }\left(j^{\prime}-j-n, p_{2} \lambda_{2} e^{-\mu_{2} \alpha} t, i, M-j-n\right) \\
& \times P_{\inf }\left(n, \lambda_{1} e^{-\mu_{1} \alpha}, j, M-j\right)
\end{aligned}
$$

According to continuous Markov chain's definition, every element of generator matrix $\mathrm{Q}$ is associated with transferring probability $p$ as exhibits:

$$
\begin{aligned}
& q_{i, j \rightarrow i, j}=-\lim _{t \rightarrow 0} \frac{1-p_{i, j \rightarrow i, j}(t)}{t}, \\
& q_{i, j \rightarrow i^{\prime} j^{\prime}}=\lim _{t \rightarrow 0} \frac{p_{i, j \rightarrow i^{\prime} j^{\prime}}(t)}{t}\left(i \neq i^{\prime} \text { or } j \neq j^{\prime}\right),
\end{aligned}
$$

where element $q_{i, j \rightarrow i, j}$ represents the speed of being static, $q_{i, j \rightarrow i^{\prime}, j^{\prime}}$ represents the transferring rate from state $(i, j)$ to state $\left(i^{\prime}, j^{\prime}\right)$ while the state in front of the arrow stands for the row and the state after the arrow stands for the column of matrix Q.

We define $K=(\lceil r * N\rceil-1) *(M+1)$, Matrix Q could also be divided into several sub-matrixes in the following form:

$$
Q=\left[\begin{array}{cc}
T & R \\
0 & 0
\end{array}\right],
$$

where $T$ is a $K \times K$ matrix, $R$ is a $K \times 1$ matrix denoting the transition rate from transient state $(i, j)$ to absorbing state Succ. Then the average delay (the average time to get to absorbing state) can be derived as $D_{d}=\int_{0}^{\infty} G_{D}(t) d t$. From work of [1], we can get that $G_{D}(t)=e \cdot \exp (T t) \cdot I$, consequently $D_{d}=e \cdot\left(-T^{-1}\right) \cdot I$, where $e$ is a vector denoting the initial state probability vector $e=[1,0,0, \ldots, 0]$ and $I$ is an all-one vector $I=[1,1, \ldots, 1]$.

\section{PERFORMANCE EVALUATION}

In this section, we quantify the performance of information dissemination delay derived from the proposed model. The parameters are set as follows, $\lambda_{1}=1 / 400$, $\lambda_{2}=1 / 1000, \mu_{1}=0.8, \mu_{2}=1, r=0.8, N=50, M=50$, $p_{1}=0.8, p_{2}=0.6$.

It can be seen from Fig. 1(a) that while the bundle size is relatively small, average delay increases slowly. However, when the bundle size is getting larger, the average delay grows exponentially. On the other hand, the average delay is also greatly prolonged by the rise of the ratio of nodes ought to be infected. Fig. 1(b) plots the average delay as a function of the number of nodes in A and B for unequal message sizes. Although $r$ is fixed, with the increase of $\mathrm{N}$ and $\mathrm{M}$, the average delay astonishingly degraded. Such a phenomenon might be attributed to the fact that each new node is a potential relay in the epidemic graph that might help in the spreading of message. Fig. 1(c) shows the impact of intra-group and inter-group forwarding willingness on dissemination delay. As intra-group connections are more probable to set up, the increase in intra-group willingness remarkably reduced the average delay, while the variance of inter-group willingness has much smaller effect on it.

\section{ACKNOWLEDGMENTS}

This work is supported by National Nature Science Foundation of China (No. 61171065, No. 61021001) and Chinese National Major Scientific and Technological Specialized Project (No. 2010ZX03004-002-02), as well as the PCSIRT and TNLIST.

\section{REFERENCES}

[1] Y. Li, P. Hui, D. Jin, L. Su, and L. Zeng. Evaluating the impact of social selfishness on the epidemic routing in delay tolerant networks. Communications Letters, IEEE, 14(11):1026-1028, 2010.

[2] J. Whitbeck, V. Conan, and M. de Amorim. Performance of opportunistic epidemic routing on edge-markovian dynamic graphs. Arxiv preprint arXiv:0909.2119, 2009. 